\title{
A Cognitive Reference Based Model for Learning Compositional Hierarchies with Whole-Composite Tags
}

\author{
Anshuman Saxena ${ }^{1,2}$, Ashish Bindal ${ }^{1}$ and Alain Wegmann ${ }^{1}$ \\ ${ }^{I}$ Systemic Modeling Laboratory, I\&C EPFL, Lausanne Switzerland \\ ${ }^{2}$ TCS Innovation Labs, Bangalore India \\ \{anshuman.saxena, ashish.bindal, alain.wegmann\}@epfl.ch
}

Keywords: Service design, Part-whole relations, Situated conceptualization, Linguistic markers, Digraph analysis.

\begin{abstract}
A compositional hierarchy is the default organization of knowledge acquired for the purpose of specifying the design requirements of a service. Existing methods for learning compositional hierarchies from natural language text, interpret composition as an exclusively propositional form of part-whole relations. Nevertheless, the lexico-syntactic patterns used to identify the occurrence of part-whole relations fail to decode the experientially grounded information, which is very often embedded in various acts of natural language expression, e.g. construction and delivery. The basic idea is to take a situated view of conceptualization and model composition as the cognitive act of invoking one category to refer to another. Mutually interdependent set of categories are considered conceptually inseparable and assigned an independent level of abstraction in the hierarchy. Presence of such levels in the compositional hierarchy highlight the need to model these categories as a unified-whole wherein they can only be characterized in the context of the behavior of the set as a whole. We adopt an object-oriented representation approach that models categories as entities and relations as cognitive references inferred from syntactic dependencies. The resulting digraph is then analyzed for cyclic references, which are resolved by introducing an additional level of abstraction for each cycle.
\end{abstract}

\section{INTRODUCTION}

A compositional hierarchy is the default organization of knowledge acquired for the purpose of specifying the design requirements of a service (Saxena and Wegmann 2012). A service seeks to influence aspects of reality through the creation of man-made artifacts. A compositional hierarchy organizes the categories observed in reality in a hierarchical manner such that the categories at the lower level contribute to the behavior exhibited by the categories at the higher level. Knowledge that reveals the composition of some observed behavior by identifying its constituent categories is, in general, useful for engineering purposes. Furthermore, a hierarchical organization of such knowledge, structures the constituent categories based on their relative strength of interactions (Simon 1962). The resulting levels correspond to the different aspects of the composition, which can either be tagged as novelty revealing composites or simply structure enforcing composites. Novelty is a subjective notion that resides in the ability of the observer to discern a conceptualization into coherent, though connected and possibly overlapping, regions in semantic space. In the context of compositional hierarchy, levels exhibiting novel properties signify strong interdependence among the descendant nodes. The inseparability of the conceptual relevance of such descendant nodes suggests that these nodes should be modeled as a unified-whole wherein the individual nodes can only be characterized in the context of the behavior of the descendant set as a whole. For an artifact to deliver desired results in a given situation, the design of the artifact must possess an amount of variety that is at least equal to the variety that the situation may present (Ashby 1964). An explicit acknowledgement of the existence of such unified-wholes as an integral part of the observed reality helps ensure that the properties associated with the unified-wholes are preserved in the target service, which, in turn, amounts to adding variety to the service specification, thereby increasing the likelihood that the service yields desired benefits. 


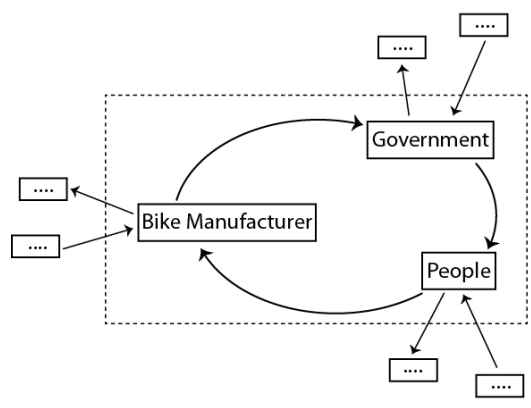

(a)

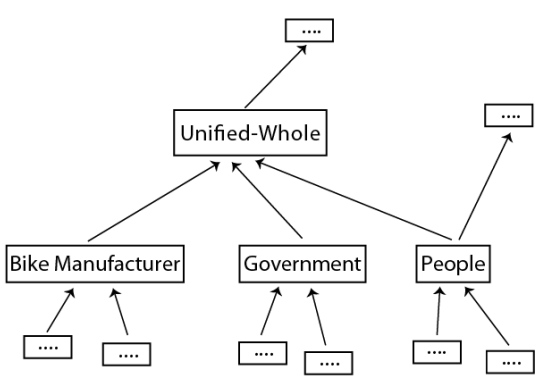

(b)

Figure 1: A compositional hierarchy extracted from the sample text $\mathcal{T}$ 'depicting the notion of unified-whole as a set of strongly interdependent categories.

For example, consider the following sample text, $\mathcal{T}$. The interdependencies between the categories occurring in this sample text and the corresponding compositional hierarchy are depicted in figure 1 .

\section{$\mathcal{T}$ : Bike manufacturers are increasingly} engaging with people to identify new bike designs. The demand for bikes has gone up. More and more people are now riding bikes. The government has improved the infrastructure by adding dedicated bike lanes for riding the bikes. People feel safe in bike lanes. Government is also encouraging bike manufacturers to increase their production by subsidizing their operations through tax waivers and easy loans. More and more people riding the bike results in a healthy society, which, in turn, lowers the cost of health care for the government.

Large amount of information about various aspects of the real world is available as natural language documents. In the context of service design, the socio-economic narrative that is most relevant for modeling the real or intended behavior of the participating actors, both human and otherwise, is very often embedded in vision papers, policy guidelines, surveys, and field-study reports (Zarri 1997). Conventional means for learning compositional hierarchies from such unstructured natural language text, interpret composition as an exclusively propositional form of part-whole relation. Propositions define a conceptualization as a set of truth-conditions that are evaluated to ascertain if a conceptualization holds in a given context. For example, a part-whole conceptualization represented in propositional form as part (wheel, bike) is considered decodable from a given text if and only if the text contains the natural language expression 'wheel is part of the bike'.
Nevertheless, not all information encoded in linguistic utterances may lend itself entirely to truthconditions based decoding (Wilson and Sperber 1993). In addition, the utterance may also contain information that is not analytically relevant to the proposition, yet equally important in invoking the perceptual experience associated with the meaning of the utterance that the proposition seeks to model. For example, to infer from the sample text $\mathrm{T}$ that the categories - Bike manufacturer, Government and People contribute to each other's behavior and, hence, constitute a unified-whole is quite challenging. The text contains no explicit mention of the unified-whole or any semantic relation that can be mapped to the semantic primitives of a partwhole relation (Winston, Chaffin et al. 1987). As a result, it is difficult to devise linguistic markers that can be used to extract such implicit compositional information based on purely propositional forms of part-whole relation.

A situated view of conceptualization (Barsalou 2009) is grounded in the perceptual experience that is associated with a category. In the context of natural language processing, it models information contained in a linguistic expression as not localized in some fixed, predetermined lexical pattern but as distributed across different aspects of the various natural language expressions constituting the discourse (Langacker 2008). The basic idea is to adopt an experientially grounded approach to conceptualization and model composition at a preconceptual level - as an embodied pattern of cognitive reference, (Rosch 1975) (Tribushinina 2008). Cognitive reference provides a generalized interpretation of composition as an interaction between two categories such that one category serves as the reference for understanding the other and that this reference has some cognitive appeal to the observer. The under specification of the conceptual relevance of the cognitive appeal is 
intentional as it allows to admit all possible aspects of the behavior that a category may exhibit.

From a natural language processing point of view, dependencies between different syntactic categories provide a natural means of extracting linguistic evidence for cognitive references. For example, prepositions represent a syntactic category that can be viewed as a semantic relation between a structure that precedes it, e.g. a verb, or a nounphrase, and another one that follows it, e.g. a nounphrase (Saint-Dizier 2006). Similarly, we can also interpret the verb relations and attributive relations like modifiers as evidence for cognitive referencing. In the case of verbs, we get greater specificity by also acknowledging the semantic role assignment done by parsers (Jackendoff 1987), (Fillmore 1968), (Dowty 1991).

We adopt an object-oriented representation (Sowa 1984) approach that models categories as entities and relations as cognitive references inferred from syntactic dependencies. The resulting digraph is then analyzed for cyclic references, which are resolved by introducing an additional level of abstraction for each cycle. Mutually interdependent set of categories are considered conceptually inseparable and assigned an independent level of abstraction in the hierarchy. Presence of such levels in the compositional hierarchy highlight the need to model these categories as a unified-whole wherein they can only be characterized in the context of the behavior of the set as a whole.

\section{CHARACTERIZING COMPOSITION}

Linguistic utterances encode two basic types of information - information about the state of affair it describes and information indicating various speech acts it intends to perform (Wilson and Sperber 1993). The first type of information is explicit in the sense that the state of affairs described in the utterance can be decoded directly from the various lexical and syntactic constructs used in the utterance. The second type of information is implicit, for example, expressions of subjectivity, which need additional knowledge support to be inferred. In this section, we present two characterizations of composition - the proposition based characterization, which operates at the linguistic level and the experientially situated characterization, which operates at the pre-linguistic level.

\subsection{Propositional Approach to Composition}

Propositional form of conceptualization is rooted in the logical tradition, which defines a conceptualization as a set of truth-conditions that are evaluated to ascertain if a conceptualization holds in a given context. In the context of natural language processing, truth-conditions correspond to the occurrence of the linguistic marker associated with the proposition. For example, a part-whole conceptualization represented in propositional form as part (wheel, bike) is considered decodable from a given text if and only if the text contains the natural language expression 'wheel is part of the bike'. The linguistic marker here is a lexical pattern comprised of named entities wheel, bike and the copula verb, part. The proposition cannot be decoded from any other natural language expression, for example, 'wheel is attached to the bike', or extended to partwhole relations between other categories, for example, 'roads are required to bike', unless additional truth-conditions are associated with the proposition. For the three expressions mentioned above to be decodable as a conceptualization of partwhole relation, the following truth conditions need to be specified as three separate linguistic markers: part (NE, NE), attach (NE, NE) and require ( $N E$, $N E$ ); where NE stands for named entities. Existing information retrieval methods try to minimize the false negatives associated with proposition based concept extraction by expanding the set of linguistic markers used to define the truth conditions of the proposition being decoded. Various automatic and semi-automatic schemes have been developed to identify linguistic markers corresponding to the different lexical and syntactic divergences (Dorr 1993) that the linguistic interpretation of the proposition may undergo. A widely used algorithm for extracting semantic relations through the use of lexico-syntactic patterns is described in (Hearst 1992).

From a knowledge organization point of view, logic based modeling of semantic relations help to structure the concepts in ways that permit automated inference making and is hence widely popular. As part of this modeling tradition, the part-whole relations limit composition to include only those interactions between categories that can be characterized along the following three dimensions whether the categories are functionally related to each other; whether the categories can exist independent of each other; and whether they are of the same type (Winston, Chaffin et al. 1987). The 
primary motivation to identify these semantic primitives of part-whole relations is to maintain transitivity as an invariant across all occurrences of part-whole relations in natural language use. Transitivity is an important logical property, in addition to antisymmetry and reflexivity, that underlies much of the inference-making in hierarchies, for example, query expansion (Nie 2003). These semantic primitives are often used to further improve the performance of decoding partwhole relations from natural language text by generating linguistic markers from some widely used keywords that convey the meaning associated with the semantic primitives underlying the propositional interpretation of part-whole relations (Girju and Moldovan 2002), (Khoo, Chan et al. 2000). For instance, 'cause' as a keyword for functional dependence, 'component' or 'part' as keywords for independence of existence and 'such as' or 'for example' as keywords for similarity of type. These keywords are then used to identify lexico-syntactic patterns either manually or semi automatically often with the aid of lexical knowledge bases like WordNet (Miller 1990).

\subsection{Situated Approach to Composition}

Traditionally the focus has been on propositional forms of knowledge thereby disregarding related information readily available within the language domain, for example, expressions of subjectivity and linguistic expressions outside the proposition (Narrog 2005). One way of interpreting implicit experiential information is to view them as encoded in semantic relations that do not have an explicit mapping to the semantic primitives associated with part-whole relations. As a result they cannot be decoded directly but can only be inferred from the larger context in which they occur. Very often this context may be distributed across several sentences. Cognitively, conceptualization is situated (Barsalou 2003). It is the reenactment of a combination of prior experiences that together simulate a perceptual experience in the form of a situation - experienced or imaginary. A simulated situation captures only one of many possible aspects of a category observed in reality. Diverse aspects of a category may get simulated across different situations. A situated view of conceptualization is an experientially grounded view of conceptualization and, in the context of language, it models information contained in a linguistic expression as not localized in some fixed predetermined lexical pattern but as distributed across different aspects of the expressions constituting a discourse (Langacker 2008). The basic idea is to adopt an experientially grounded approach to conceptualization and model composition as a pre-conceptual embodied pattern.

Simulating perceptual experience from these modal states is then an exercise of inferring and/or composing a situation. The multi-modal experience that the situation represents is reenacted at the different modal systems thereby simulating an experience of being in that specific situation. Such multi-modal simulation based model of conceptualization highlights the situated nature of concepts and is referred to as situated conceptualization (Barsalou 2009). Such situation specific inferences are, in principle, motivated by the theory of situation semantics, where logical inference is optimized when performed in the context of specific situations (Barwise and Perry 1983).

\subsubsection{Cognitive Reference as an Embodied Pre-linguistic Structure of Composition}

A pre-linguistic structure of conceptualization refers to the organization of knowledge at a level of abstraction that is higher than the linguistic level, where organization is limited to explicitly stated propositions. The knowledge available at such higher levels of abstraction is experiential in nature, with both explicit and implicit information encoded in the linguistic utterance contributing to the perception of the experience. Various cognitive constructs have been proposed to motivate the organization of knowledge at the pre-linguistic level. These include the notion of force dynamics (Talmy 1988), image schemas (Lakoff and Johnson 2003), construals (Langacker 1987), mental spaces (Fauconnier 1994) and reference point constructions (Langacker Ronald 1993).

Amongst these the notion of cognitive reference point (CRP) construction lends itself naturally to the modeling of composition at the pre-linguistic level. CRP is the cognitive act of referring one entity by invoking another (Rosch 1975). A CRP models composition to include not only propositional forms of part-whole relation but any relation, distributed or local, that establishes a link between two categories such that link has some conceptual relevance and is asymmetric in nature. The asymmetry requirement of the link restricts the interpretation of CPR to only those relations, which clearly distinguish foreground information (focal category) from background information (contextual category) and protects it 
from the risk of degenerating to any meaningful relation between categories. Meanwhile, the under specification of the conceptual relevance of the cognitive appeal is quite useful for modeling composition as it allows to admit all possible aspects of the behavior that a category may exhibit.

\subsubsection{Interpreting novelty from circular cognitive referencing}

The notion of novelty can be explained both ontologically and epistemologically (Bunge 2004). From an ontological point of view, novelty is said to occur only when there is explicit knowledge about a new category and adequate information to verify the associated novel property using some truthconditional formulation. In the context of this work, we follow an epistemic interpretation of novelty as patterns of association, which only indicate the occurrence of novelty and provide no additional information that could help reify the ontological status of the indicated novelty.

Cognitive reference provides a generalized interpretation of composition as an interaction between two categories such that one category serves as the reference for understanding the other and that this reference has some cognitive appeal to the observer. As a result, mutually interdependent set of categories are considered conceptually inseparable and assigned an independent level of abstraction in the hierarchy. Presence of such levels in the compositional hierarchy highlight the need to model these categories as a unified-whole wherein they can only be characterized in the context of the behavior of the set as a whole.

\section{APPROACH}

Instances of cognitive reference from text can be interpreted from dependencies between lexical elements in a sentence. The fundamental notion of dependency is based on the idea that the syntactic structure of a sentence consists of binary asymmetrical relations between the lexical elements of a natural language expression (Tesniere 1959). Dependency types commonly used in dependency parsers include surface-oriented grammatical functions, such as subject, object, modifiers, and a set of more semantically oriented role types, such as agent, patient, and goal (Nivre 2005). Semantic roles are theme revealing relations that express the role that a noun phrase plays with respect to the action or state described by a sentence (Jackendoff 1987), (Dowty 1991). When these roles are defined exclusively in relation to the sub-categorization frame of the verb they are referred to as case roles (Fillmore 1968).

We use the Stanford dependency parser for English language text (de Marneffe, Maccartney et al. 2006). Following the terminology used in the Stanford dependency manual, a dependency relation holds between a governor and a dependent and is represented

as: dependency (governor, dependent). Each dependency connection, in principle, links a superior term and an inferior term. The superior term receives the name governor and the inferior the name dependent. The superior/inferior characterization for a pair of words is based on different morphological, syntactic and semantic considerations. In the context of this work, the interest is more to characterize superior/inferior from a cognitive reference point of view - superior as the one in the foreground (focal) and inferior as the one in the background (context). The background word contributes to the understanding of the word in the foreground. As mentioned in (Langacker 1994), the structural syntax based dependency framework and the cognitive reference framework have substantial similarity. The acknowledgement of the underlying similarity encourages us to re-interpret dependency relations between lexical items from a cognitive reference perspective.

The Stanford dependency manual (De Marnee and Manning 2011) lists 53 grammatical relations. Table 1 lists the dependencies considered in this work and their re-interpretation as focal and contextual categories.

\begin{tabular}{lll}
\hline Type & Syntactic dependency & Cognitive reference \\
\hline Verb & $* \operatorname{obj}(A, B)$, agent(A,B) & A(focal), B(contextual) \\
& $*_{\text {subj(A,B) }}$ & A(contextual), B(focal) \\
Preposition & prep*(A,B) & A(focal), B(contextual) \\
Attribute Modifiers & amod(A,B) & A(focal), B(contextual) \\
\hline
\end{tabular}

Table 1: Interpreting syntactic dependencies as cognitive references. 


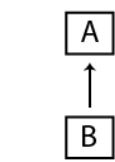

*obj(A, B)

agent $(A, B)$

${ }^{*} \operatorname{prep}(A, B)$

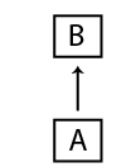

${ }^{*} \operatorname{subj}(A, B)$

(b)

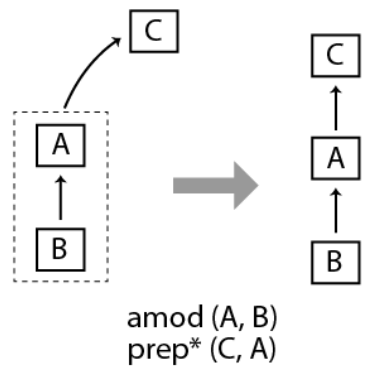

(c)

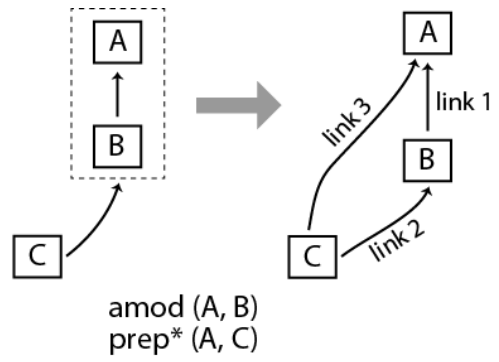

(d)

Figure 2: A visual depiction of the mappings from syntactic dependencies to cognitive reference

Most dependencies can be interpreted directly as a cognitive reference link, with the governor as the focal category and the dependent as the context. For subject dependencies, it is the other way round: governor as the context and dependent as the focal category. This is due to the interpretation of verb as a link between its different arguments - the object qualifying the meaning of the verb and subject being qualified by verb as providing the context in which the subject is being referred. A visual depiction of the cognitive reference links inferred from syntactic dependencies is provided in figure 2. A special pattern resulting from a combination of attributive and prepositional dependencies is worth mentioning. The case where the focal categories are different, e.g. figure 2(c), the combination of attributive and prepositional qualifiers can be organized as a unipath hierarchy, which can be seen as context refinement. The case where the focal categories are the same results in a multipath-hierarchy as the focal category can be interpreted in multiple contexts, figure $2(\mathrm{~d})$.

Consider the following sentence:

\section{$S:$ The government has improved the infrastructure} by adding dedicated bike lanes for riding the bikes.

The dependencies generated by the Stanford parser for $S$ are depicted as a graph in figure 3. This visualization is obtained using a freely available plug-in, DependenSee, from the Stanford natural language processing group website (Group 2012). It is important to note that the cognitive reference point relation links lexical elements with binary asymmetrical relations as a result each sentence can be depicted as a directed acyclic graph, DAG. As explained earlier, the only semantics associated with this link is encoded in its direction - the source being the constituent category and the destination the focal category. The DAG depicting the cognitive reference links embedded in the sentence $S$ is shown in figure 4.

The DAG for each sentence in the text is merged by only admitting one node per category. Conceptually this amounts to making explicit the implicit connections in the text. The resulting graph is directed but not necessarily acyclic. The cycles in the digraph correspond to interdependence between categories. The digraph representing the cognitive references between categories described in $\mathcal{T}$ is shown in figure 5 .

The cognitive reference digraph is then analyzed for cycles by identifying the strong components of the digraph. A strongly connected component of a digraph is a maximal set of vertices in which there is a path from any one vertex to any other vertex in the set (Tarjan 1972). The algorithm used to identify the strongly connected components of the digraph is due to KosarajuSharir and described in detail in (Sedgewick 2011).

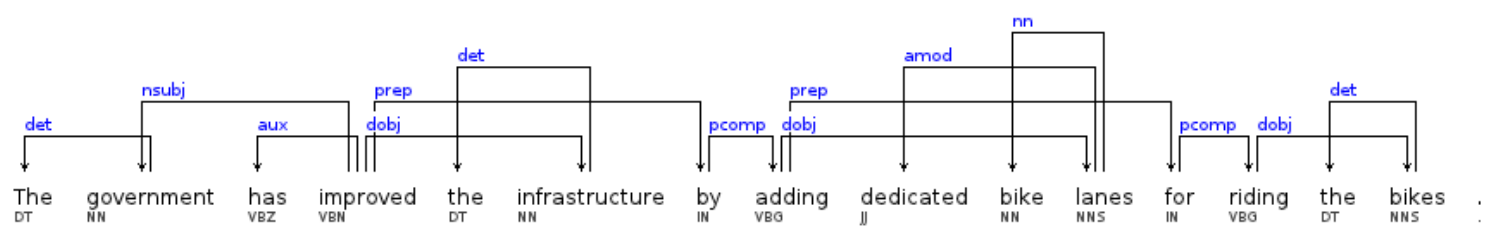

Figure 3: Dependency graph visualization of $S$ 


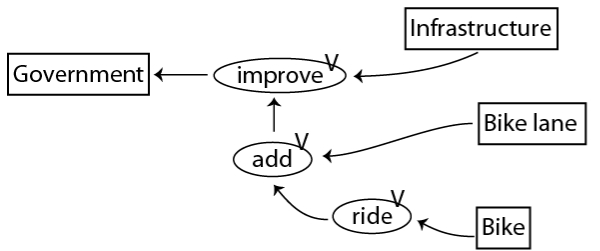

(a)

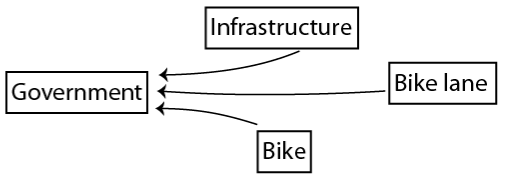

(b)

Figure 4: Cognitive reference graph visualization of $S$

\section{CONCLUSIONS}

Data mining is defined as “...the analysis of (often large) observational data sets to find unsuspected relationships and to summarize the data in novel ways that are both understandable and useful to the data owner" (Hand, Smyth et al. 2001). Nevertheless, efforts to find unsuspected relationships from data and their use in formulating new hypothesis should not be interpreted as the absence of any initial hypothesis, which in the first place guides one to find such unsuspected relationships. For example, the use of distributional hypothesis that assumes terms to be similar to the extent to which they share similar linguistic contexts (Harris 1968). In this case what is unknown is the nature of similarity and its relationship to different patterns of linguistic context. Communicating this work to the data mining community is equally relevant as it presents a cognitive model of composition, which can be used as a starting point for developing new data mining schemes realize this model in a computational setting.

The primary purpose of this work is to suggest an alternate conceptualization of composition and show how it can be more rewarding by making it easy to identify novel aspects of observed reality. From a relevance point of view, the work was motivated based on its usability in a very concrete

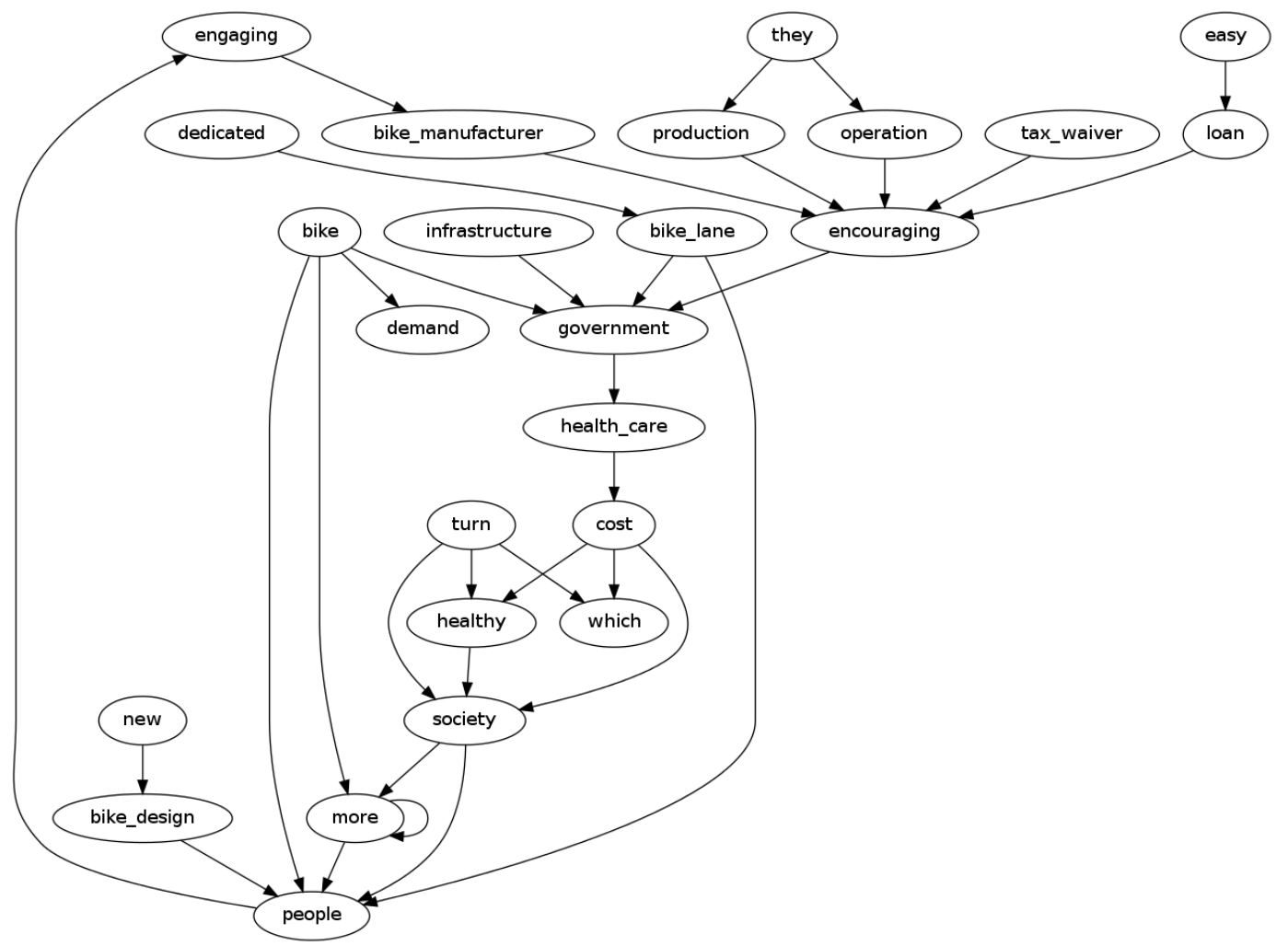

Figure 5: Cognitive reference revealing digraph visualization of $\mathcal{T}$ 


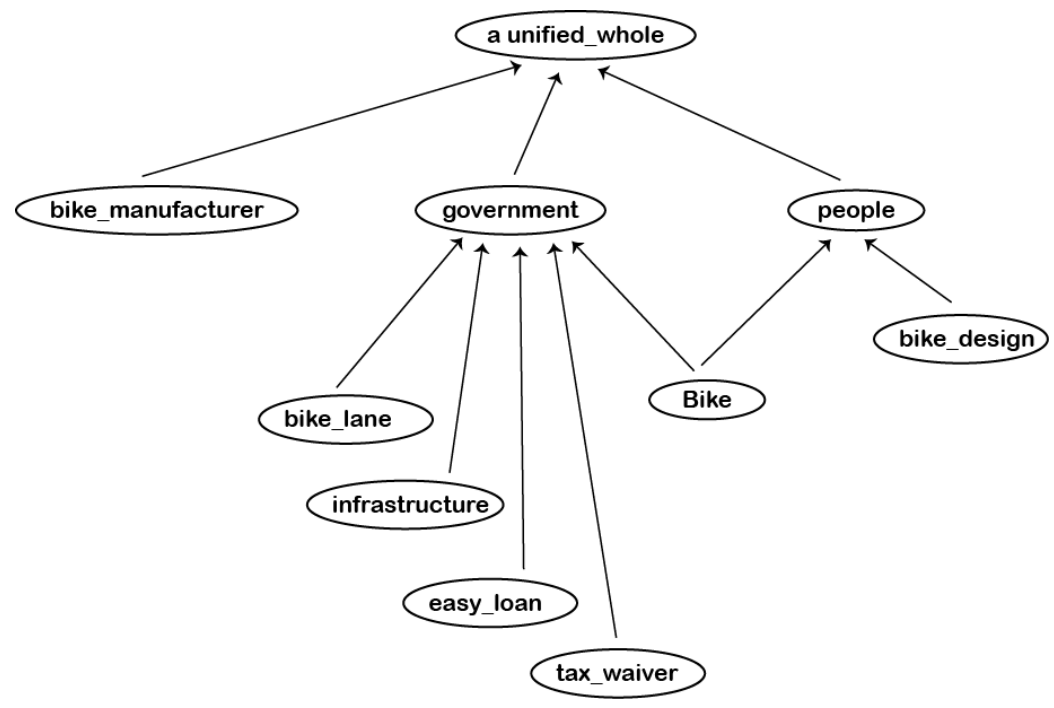

Figure 6: Compositional hierarchy representation of $\mathcal{T}$ depicting integrated-wholes

domain that of service design specification by linking novelty to the notion of requisite variety, thereby making service design conscious of the need to anticipate the operating environment conditions and account for them by including enough flexibility in their design. The current exposition is, however, limited in its scope to only identify the existence of novelty and not to provide any conceptual interpretation of the novelty of the unified-wholes.

An important future work in this regard is to apply this model of extracting compositional hierarchies to diverse text samples and study the extent to which it is able to detect cognitive reference cycles. Based on our experimentation, there could be situations where the cognitive interdependence may not be detectable as a complete cycle and some threshold based connectivity measure might help further reduce the false negatives associated to identify unified-whole in compositional hierarchies.

\section{REFERENCES}

Ashby, W. R. (1964). Introduction to Cybernetics, Methuen.

Barsalou, L. (2009). "Simulation, situated conceptualization, and prediction." Philosophical Transactions of the Royal Society B: Biological Sciences 364(1521): 1281-1289.

Barsalou, L. W. (2003). "Situated simulation in the human conceptual system." Language and Cognitive Processes 18(5): 513-562.
Barwise, J. and J. Perry (1983). Situations and attitudes. Cambridge, MA, MIT Press.

Bunge, M. (2004). Emergence and Convergence: Qualitative Novelty and the Unity of Knowledge, University of Toronto.

De Marnee, M.-C. and C. Manning (2011). Stanford typed dependencies manual.

de Marneffe, M. C., B. Maccartney, et al. (2006). Generating Typed Dependency Parses from Phrase Structure Parses. LREC.

Dorr, B. J. (1993). Machine Translation: A view from the lexicon. Cambridge, Massachusetts, The MIT Press.

Dowty, D. (1991). "Thematic Proto-Roles and Argument Selection." Language 67(3): 547-619.

Fauconnier, G. (1994). Mental Spaces: Aspects of Meaning Construction in Natural Language, \{Cambridge University Press\}.

Fillmore, C. J. (1968). The Case for Case. Universals in Linguistic Theory. E. H. Bach, Robert. New York, Holt, Rinehart, and Winston.

Girju, R. and D. I. Moldovan (2002). Text Mining for Causal Relations. Proceedings of the Fifteenth International Florida Artificial Intelligence Research Society Conference, AAAI Press: 360-364.

Group, S. N. L. P. (2012). "The Stanford parser: A statistical parser." Retrieved October 07, 2012, from http://nlp.stanford.edu/software/lex-parser.shtml.

Hand, D. J., P. Smyth, et al. (2001). Principles of data mining, MIT Press.

Harris, Z. (1968). Mathematical structures of language, Interscience Publishers. 
Hearst, M. A. (1992). Automatic acquisition of hyponyms from large text corpora. Proceedings of the 14th conference on Computational linguistics - Volume 2. Nantes, France, Association for Computational Linguistics: 539-545.

Jackendoff, R. (1987). "The Status of Thematic Relations in Linguistic Theory." Linguistic Inquiry 18(3): 369411.

Khoo, C. S. G., S. Chan, et al. (2000). Extracting causal knowledge from a medical database using graphical patterns. Proceedings of the 38th Annual Meeting on Association for Computational Linguistics. Hong Kong, Association for Computational Linguistics: 336-343.

Lakoff, G. and M. Johnson (2003). Metaphors We Live By, University Of Chicago Press.

Langacker, R. (1987). Foundations of Cognitive Grammar. Vol. 1: Theoretical Prerequisites. Stanford, Stanford University Press.

Langacker Ronald, W. (1993). Reference-point constructions. Cognitive Linguistics (includes Cognitive Linguistic Bibliography). 4: 1.

Langacker, R. W. (1994). "Structural Syntax: The View from Cognitive Grammar." Semiotique 6/7: 69-84.

Langacker, R. W. (2008). Cognitive Grammar: A Basic Introduction. New York, Oxford University Press.

Miller, G. A. (1990). "Nouns in WordNet: A Lexical Inheritance System." International Journal of Lexicography 3(4): 245-264.

Narrog, H. (2005). "On defining modality again." Language Sciences 27(2): 165-192.

Nie, J.-Y. (2003). "Query expansion and query translation as logical inference." J. Am. Soc. Inf. Sci. Technol. 54(4): 335-346.

Nivre, J. (2005). Dependency Grammar and Dependency Parsing.

Rosch, E. (1975). "Cognitive reference points." Cognitive Psychology 7(4): 532-547.

Saint-Dizier, P. (2006). Introduction to the Syntax and Semantics of Prepositions

Syntax and Semantics of Prepositions. P. Saint-Dizier, Springer Netherlands. 29: 1-25.

Saxena, A. B. and A. Wegmann (2012). From Composites to Service Systems: The Role of Emergence in Service Design. IEEE International Conference on Systems, Man, and Cybernetics. Seoul, Korea.

Sedgewick, R. (2011). Algorithms. Boston, MA, AddisonWesley.

Simon, H. A. (1962). "The Architecture of Complexity." Proceedings of the American Philosophical Society 106(6): 467-482.
Sowa, J. F. (1984). Conceptual structures: Information processing in mind and machine. Reading, MA, Addison-Wesley.

Talmy, L. (1988). "Force Dynamics in Language and Cognition." Cognitive Science 12(1): 49-100.

Tarjan, R. (1972). "Depth-First Search and Linear Graph Algorithms." SIAM Journal on Computing 1(2): 146160.

Tesniere, L. (1959). Elements de syntaxe structurale. Paris, Klincksieck.

Tribushinina, E. (2008). Cognitive reference points: semantics beyond the prototypes in adjectives of space and colour. Doctoral Thesis, Leiden University.

Wilson, D. and D. Sperber (1993). "Linguistic form and relevance." Lingua 90(1-2): 1-25.

Winston, M., R. Chaffin, et al. (1987). "A Taxonomy of Part-Whole Relations." Cognitive Science 11(4): 417444.

Zarri, G. (1997). Conceptual modelling of the "meaning" of textual narrative documents. Foundations of Intelligent Systems. Z. Ras and A. Skowron, Springer Berlin / Heidelberg. 1325: 550-559. 